\title{
L'UTILISATION DE L'ANTIPYRINE POUR LA MESURE IN VIVO DE L'EAU TOTALE DU CORPS CHEZ LES OVINS
}

PAR

\section{B.-L. DUMONT}

Station de Recherches sur 1'Élevage C.N.R.Z., Jouy-en-Josas ( $\left.{ }^{1}\right)$.

$I_{1}$ a détermination in vivo de la masse hydrique corporelle a provoqué, depuis quelques années, d'importantes recherches. La méthode originale de SobERMan et coll. (I) utilisant l'antipyrine, a été appliquée à diverses espèces domestiques (chiens, lapins, bovins, porcins) par différents auteurs, qui ont précisé, dans chaque cas particulier, les détails de sa réalisation : dose à injecter, horaires des prélèvements.

Un seul travail a fait mention, jusqu'ici, des possibilités d'utilisation de cette méthode chez les ovins (2), sans toutefois indiquer les règles pratiques à observer et sans en discuter la précision.

Il nous a paru utile, avant d'utiliser systématiquement cette méthode dans notre Station, d'en préciser les conditions d'emploi et, éventuellement, les limites d'utilisation dans cette espèce.

\section{Technique expérimentale}

Cette étude a porté sur 33 animaux de race Texel, âgés de 4 mois à 3 ans $\mathrm{I} / 2$, et pesant entre 42 et $90 \mathrm{~kg}$.

Sur ces animaux, I3 furent uniquement utilisés pour l'examen de la diffusion de 1'antipyrine dans les divers tissus.

Les injections d'antipyrine (faites avec une seringue calibrée) et les prises de sang furent pratiquées à l'une des jugulaires. Les sangs et les tissus furent analysés suivant les mêmes techniques que celles précédemment décrites chez le porc (3).

\section{Résultats}

\section{Vitesse de disparition}

L'antipyrine disparaît assez rapidement du sang après son introduction dans l'organisme, comme l'indique la figure I, représentant les

(1) Ce travail a été réalisé avec la collaboration technique de Mile Éléonora IHudzik et de M. Jean WERFR. 
variations de la concentration du plasma en antipyrine, en fonction du temps après injection.

Le pourcentage de disparition horaire est, en moyenne, de:

54,35 p. Ioo entre la $I^{\text {re }}$ et la $2^{\mathrm{e}}$ heure

54,85 p. Ioo entre la $2^{\mathrm{e}}$ et la $3^{\mathrm{e}}$ heure

49,75 p. Ioo entre la $3^{\text {e }}$ et la $4^{\mathrm{e}}$ heure.

Cette vitesse de disparition, inférieure à celle observée chez le porc, est néanmoins élevée et dépasse sensiblement celle enregistrée chez les bovins (4).
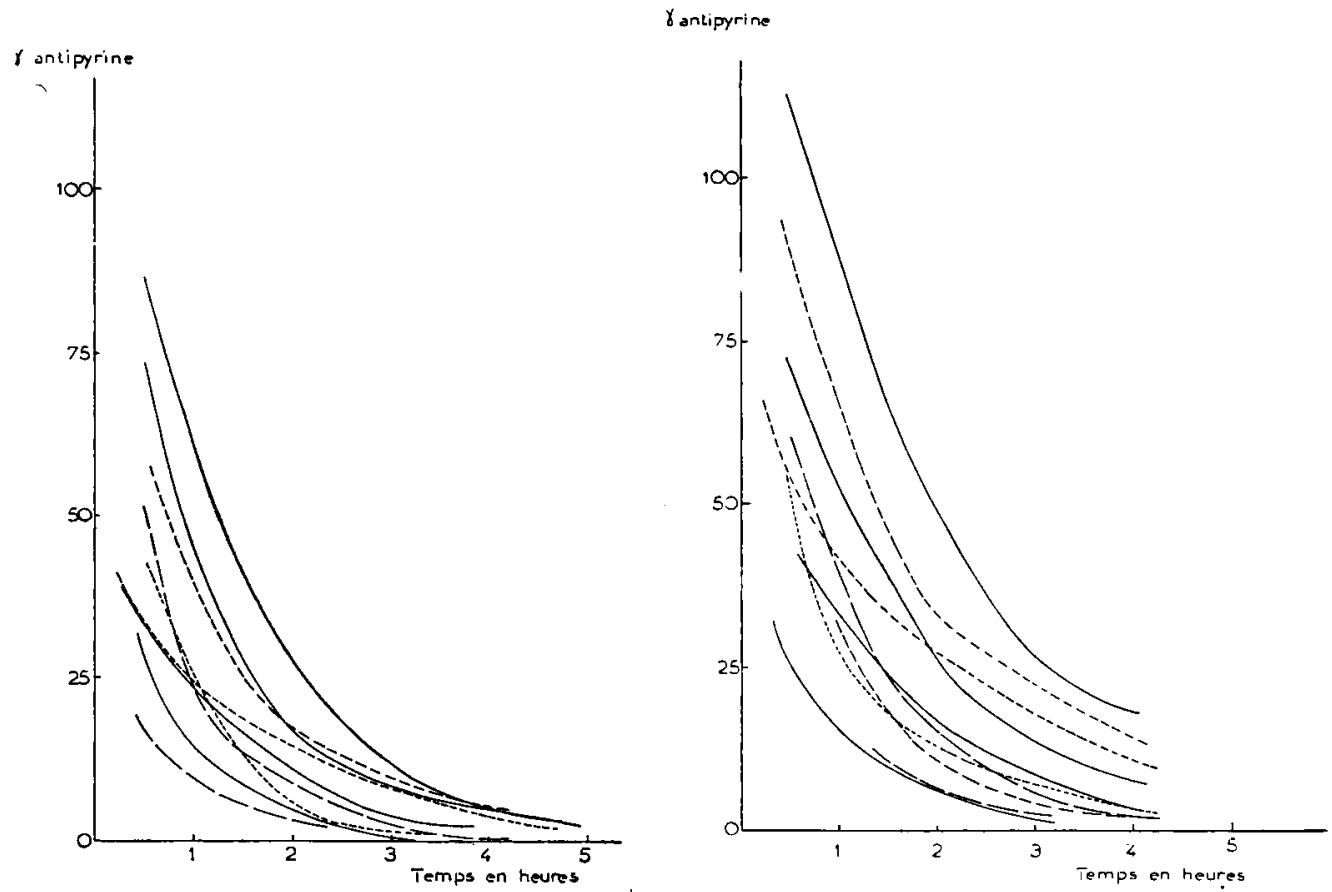

Fig. t. - Variation de la concentration du plasma en antipyrine en fonction du temps après injection.

La dose ne semble pas avoir d'influence sur la vitesse d'élimination, du moins dans les limites des doses injectées ici (de I,5 g à $8 \mathrm{~g}$ pour rookg de poids vif).

Des variations individuelles importantes sont à noter (valeurs extrêmes du pourcentage de disparition horaire : 30,3 p. Ioo-88 p. IOo), plus importantes que celles constatées chez les bovins et les porcins, mais eu même ordre que celles observées chez le chien (5).

\section{Quantité d'eau calculée}

La figure 2 représente la répartition des quantités d'eau calculées en fonction du poids vif. Les pourcentages correspondants d'eau corporelle vont de 33,5 à $5^{8,2} \mathrm{p}$. Ioo du poids vif. 


\section{Discussion}

\section{a) Horaire des prélèvements}

Quarante minutes après l'injection, les concentrations du plasma en antipyrine fournissent des points qui sont alignables sur la droite d'élimination, ce qui traduirait une diffusion corporelle très rapide de la substance. L'ajustement à la droite est possible jusqu'à 3 heures après injection. Au-delà, il n'est possible qu'après injection de doses assez fortes (égales ou supérieures à $4 \mathrm{~g}$ pour Ioo $\mathrm{kg}$ de poids vif) et lorsque le pourcentage de disparition de la substance est faible: moins de $40 \mathrm{p}$. Ioo.

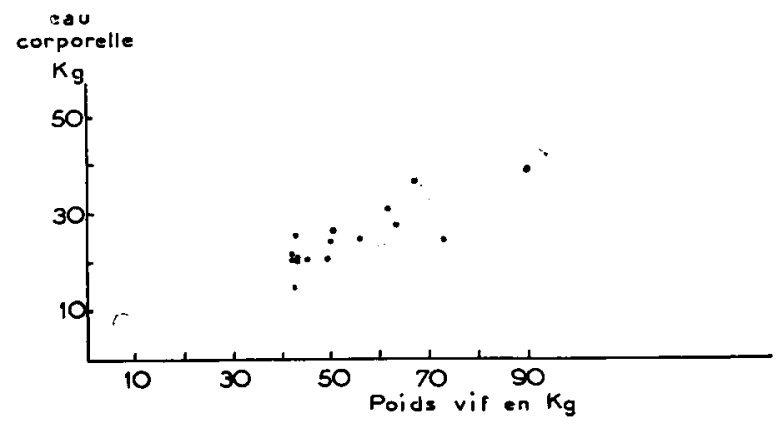

Fig. 2.

Compte tenu de ces observations, les conditions de la réalisation de la mesure de l'espace de diffusion de l'antipyrine sont les suivantes :

injecter une dose de l'ordre de $4 \mathrm{~g}$ pour roo $\mathrm{kg}$ de poids vif,

pratiquer 4 , ou mieux 5 prélèvements de sang, entre 50 minutes et 3 heures après injection.

\section{b) Précision de la mesure}

L'ajustement à la droite d'élimination des points expérimentaux autorise souvent plusieurs interprétations et peut fournir, de la valeur de la concentration au temps o, différentes valeurs, comprises entre deux valeurs extrêmes, d'autant plus espacées que la variabilité des différentes valeurs obtenues au cours de l'essai par rapport aux valeurs théoriques de la droite moyenne d'élimination, est elle-même plus grande.

La précision des mesures est fonction, pour une bonne part, comme nous l'avons vu chez le porc, de la vitesse d'élimination de la substance. Et la méthode présente d'autant plus d'intérêt que la vitesse d'élimination est plus faible.

Le tableau I indique les écarts trouvés entre les valeurs extrêmes et la valeur moyenne, exprimés en p. Ioo de cette valeur moyenne: 
La moitié des résultats donne un pourcentage inférieur à $2 \mathrm{p}$. Ioo.

La précision obtenue dans la mesure de l'espace de diffusion de l'antipyrine chez les ovins est donc, dans l'ensemble, nettement supérieure à celle obtenue chez les porcins.

Tableau I

\begin{tabular}{l|c|c|c|c|c|c}
\hline \hline & $0-1 \%$ & $\mathrm{I}-2 \%$ & $2-3 \%$ & $3-+\%$ & $+-5 \%$ & $>5 \%$ \\
\hline Nombre d'observations & 7 & 3 & 2 & 0 & 2 & 6 \\
\hline
\end{tabular}

Ce fait résulte, non seulement d'une vitesse de disparition plus faible, mais aussi d'une moins grande variabilité des valeurs expérimentales par rapport aux valeurs de la droite théorique d'élimination.

\section{c) Diffusion de l'antipyrine}

L'étude de la diffusion corporelle a porté sur 5 tissus : foie, coeur, poumons, diaphragme et graisse périrénale. Pour chacun, la détermination de l'eau et le dosage de l'antipyrine permit d'établir la valeur du rapport de diffusion:

$$
r=\left(\frac{\text { concentration tissu en antipyrine/eau tissu }}{\text { concentration plasma en antipyrine/eau plasma }}\right)
$$

Ce rapport est de I lorsque la diffusion est homogène.

Pour les différents tissus, ce rapport atteint très vite, après injection (40 minutes), sa valeur maximum, ce qui confirme une diffusion rapide de la substance. La valeur moyenne des rapports pour chaque tissu, une fois la diffusion terminée, diffère plus ou moins sensiblement de I. La diffusion la plus homogène est observée pour le muscle $(\bar{x}=0,85)$. Les tissus viscéraux et notamment le poumon, présentent des rapports de diffusion inférieurs $(\bar{x}$ poumon $=0,62)$. Pendant la période d'élimination, les différents tissus perdent inégalement leur antipyrine, le tissu musculaire étant le seul à conserver constant son rapport de diffusion pendant assez longtemps (jusqu'à 2 heures après injection). De ces résultats, i1 semblerait que la validité d'une diffusion uniforme de la substance ne soit pas parfaitement établie.

On peut arguer d'un manque de précision de la méthode d'extraction de l'antipyrine des tissus, telle que l'a proposée Brodie (6). Des essais de surcharge de tissu en antipyrine nous ont montré que le pourcentage de recouvrement de la substance est variable et peut s'écarter jusqu'à 20 p. Ioo de la valeur théorique $\left({ }^{1}\right)$.

(1) La surcharge des tissus était réalisće par addition au milieu d'hydrolyse des tissus (provenant d'animaux n'ayant pas reçu d'antipyrine), d'une quantité connue d'antipyrine. 
I1 n'en reste pas moins que, même compte tenu ae ce fait, les différences observées entre tissus, au cours de la période d'élimination, indiquent que les valeurs du rapport de diffusion varient au cours de cette période. Cette observation met en cause la constance de la diffusion et partant, l'intérêt de l'antipyrine dans la mesure de l'eau totale chez les ovins. Le même phénomène avait déjà été observé chez le porc. On doit noter que, mises à part ces observations, ce fait n'a jamais été enregistré dans les autres espèces. L,e nombre de références sur la diffusion de l'antipyrine dans les tissus est d'ailleurs faible (I-5) et n'intéresse, pour l'homme et le chien, que quelques dosages.

Il y aurait lieu de revoir, dans chaque espèce et pour les différents tissus, la variation du rapport de diffusion avant de se prononcer - de façon définitive - sur la valeur du concept de la parfaite diffusibilité de l'antipyrine. Les dosages devraient être pratiqués sur des fractions importantes des tissus les plus importants du corps.

\section{Résumẻ}

L'étude de la diffusion de l'antipyrine chez les ovins a précisé les conditions pratiques de la réalisation de cette mesure, dans les meilleures conditions. La dose à injecter est de $4 \mathrm{~g}$ par $\mathrm{I}$ oo $\mathrm{kg}$ de poids vif. Les prises doivent être effectuées entre 50 minutes et 3 heures après injection. I a vitesse de disparition est de $54 \mathrm{p}$. Ioo pendant les $2^{\mathrm{e}}$ et $3^{\mathrm{e}}$ heures après l'injection.

La précision de la méthode est discutée, ainsi que la validité de l'égalité de la diffusion de cette substance dans les différents tissus.

\section{RÉFÉRENCES BIBLIOGRAPHIQUES}

(I) Sobermai (R.), Brodie (R. B.), Lévy (B. B.), Axelrod (J.), HollanDER (V.), STEELE (J. M.). - J. Biol. Chem., 179, 3I-4I, I949.

(2) $\operatorname{Hix}($ E. L.), Evans (L. E.), Underbjerg (G. K. L.). - J. Anim. Sci., 12, 459-73, I953.

(3) Dumont (B. L.). - Ann. Zootechnie, IV, 305-3I3, I955.

(4) Kraybili (H. F.), Hankins (O. G.), Bitter (H. L.). - J. of Appl. Physiol., 3 (II), 68I-89, I95I.

(5) Herrold (M.), Sapirstein. - Proc. Exp. Biol. Med., 79-4I9, I952.

(6) Brodie (B. R.), Axelrod (J.), Soberman (R.), Lévy (B.). - J. Biol. Chem., 179, 25-30. I949. 